\title{
Microstrip Low-Pass Elliptic Filter Design Based on Implicit Space Mapping Optimization
}

\author{
Saeed Tavakoli, Mahdieh Zeinadini, Shahram Mohanna \\ Faculty of Electrical and Computer Engineering, the University of Sistan and Baluchestan, Zahedan, Iran \\ E-mail:Tavakoli@ece.usb.ac.ir \\ Received February 21, 2010; revised March 27, 2010; accepted April 28, 2010
}

\begin{abstract}
It is a time-consuming and often iterative procedure to determine design parameters based on fine, accurate but expensive, models. To decrease the number of fine model evaluations, space mapping techniques may be employed. In this approach, it is assumed both fine model and coarse, fast but inaccurate, one are available. First, the coarse model is optimized to obtain design parameters satisfying design objectives. Next, auxiliary parameters are calibrated to match coarse and fine models' responses. Then, the improved coarse model is re-optimized to obtain new design parameters. The design procedure is stopped when a satisfactory solution is reached. In this paper, an implicit space mapping method is used to design a microstrip low-pass elliptic filter. Simulation results show that only two fine model evaluations are sufficient to get satisfactory results.
\end{abstract}

Keywords: Implicit Space Mapping Optimization, Microstrip Low-Pass Elliptic Filter, Surrogate Model

\section{Introduction}

Considering the development of computer-aided design methods, optimization has become a widely used technique in design of microwave circuits. A typical design problem is to choose the design parameters to get the desired response. The space mapping (SM), introduced in [1], is a powerful technique to optimize complex models. The aim of this technique is to make a shortcut using a cheaper but less accurate model, coarse model, to gain information about the optimal parameter setting of the expensive but accurate model, fine model. To obtain the optimal design for the fine model, the SM establishes a mapping between the parameters of the two models iteratively $[1,2]$. In some cases, this mapping is not explicit and it is hidden in the coarse model. The implicit space mapping (ISM) [3], described below, addresses this issue.

First, the coarse model is optimized to obtain design parameters satisfying the design objectives. Second, an auxiliary set of parameters in the coarse model, which always remain fixed in the fine model, is calibrated to match coarse and fine models' responses. This step is known as the parameter extraction step. Examples of the auxiliary parameters are physical parameters, such as relative dielectric constant, and geometrical parameters, such as substrate height. The coarse model with updated values of auxiliary parameters is known as the surrogate, calibrated coarse, model. Considering the re-calibrated auxiliary parameters fixed, then, the calibrated coarse model is re-optimized to obtain a new set of design parameters. These design parameters are given to the fine model to evaluate its performance [4]. The design procedure is stopped when a satisfactory solution is reached.

In this paper, an optimization procedure based on ISM technique is applied to a microstrip low-pass elliptic filter. Agilent ADS and ADS Momentum [5] are employed to simulate coarse and fine models, respectively.

\section{Implicit Space Mapping Approach}

The design objective is to calculate an optimal solution for the fine model, as follows

$$
x_{f}^{*}=\arg \min _{x_{f}} \Omega\left(R_{f}\left(x_{f}\right)\right)
$$

where $\Omega$ is a suitable objective function. The fine model's response, $R_{f}$, is, for example, $\left|S_{11}\right|$ at selected frequency points. $x_{f}{ }^{*}$ is the optimal fine model parameters to be determined. It can be found using the following iterative procedure

$$
x_{f}^{k+1}=\arg \min _{x_{f}} \Omega\left(R_{s}\left(x_{f}, p^{K}\right)\right)
$$

where $R_{s}$ refers to the surrogate model's response. To 
solve Equation (1), a two-step procedure is employed. In the first step, the auxiliary parameters are calibrated so that the surrogate and fine models' responses become similar enough. The auxiliary parameters are calculated using the following equation

$$
p^{k}=\arg \min _{P}\left\|R_{f}\left(x_{f}{ }^{K}\right)-R_{s}\left(x_{f}{ }^{K}, p\right)\right\|
$$

where $p^{0}$ refers to the initial auxiliary parameters. Considering the re-calibrated auxiliary parameters fixed, then, the new surrogate model is re-optimized to obtain a new set of design parameters, $x_{f}^{\text {new }}$, in the second step. If the fine model's response for this new set of design parameters satisfies the design specifications, the algorithm is stopped. Otherwise, it re-calculates the auxiliary parameters for the current design parameters $[4,6]$.

\section{Microstrip Low-Pass Elliptic Filter}

Low-pass filters are components, which are used to eliminate unwanted harmonics. Low-pass elliptic filters can provide a fairly sharp cut-off frequency [7]. In this paper, ISM technique is applied to the optimization problem of a low-pass elliptic filter with a cut-off frequency of 7 GHz. The structure of this filter is illustrated in Figure 1.

The coarse model is composed of empirical models of simple microstrip elements, as shown in Figure 2. The design specifications are as follows:

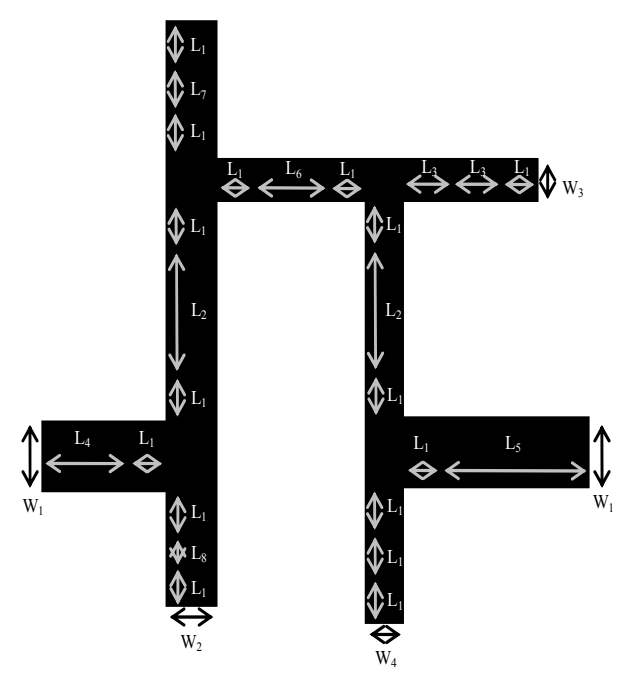

Figure 1. Microstrip low-pass elliptic filter structure.

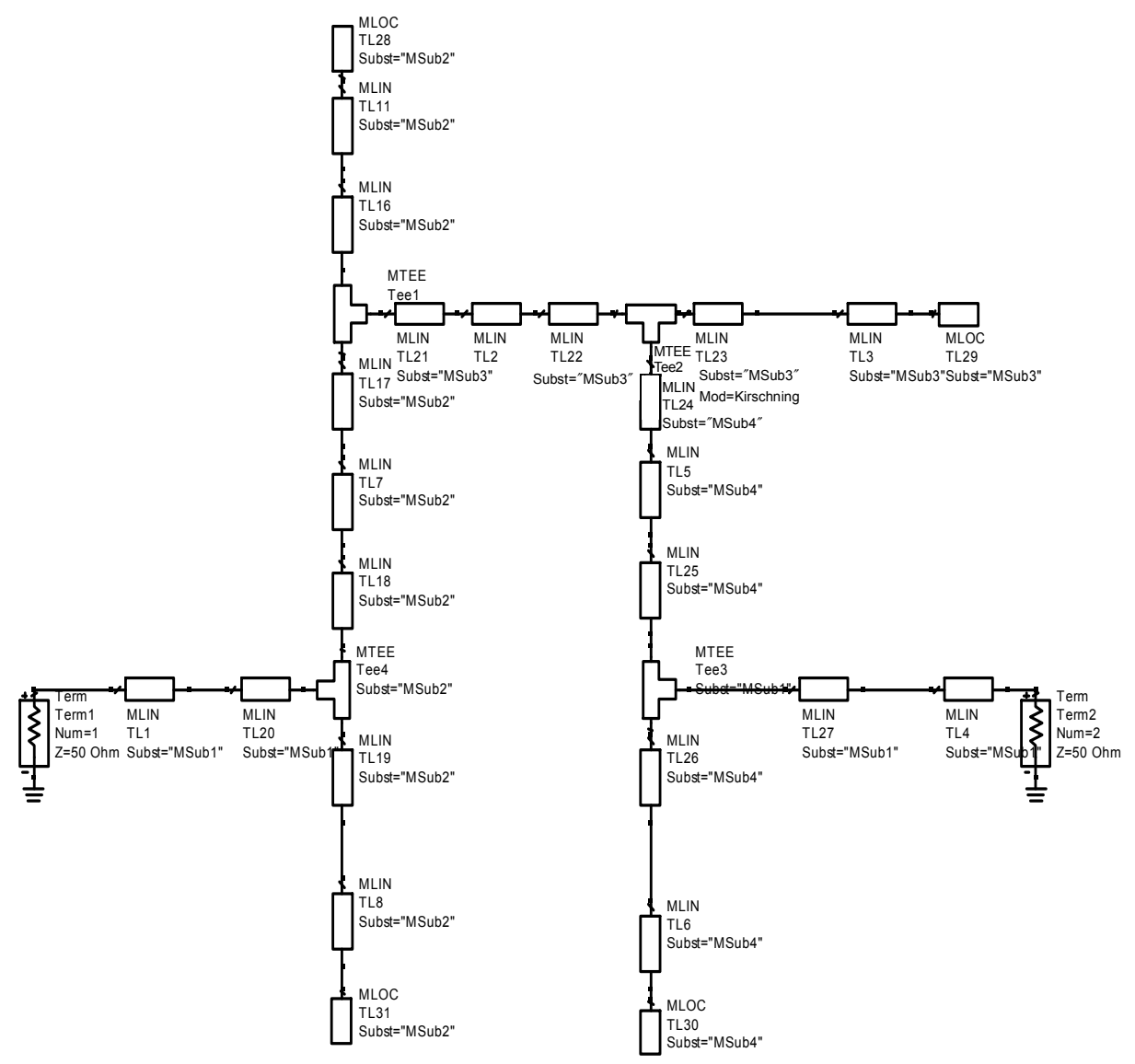

Figure 2. Coarse model simulated by ADS. 
$|\mathrm{S} 12| \geq 0.964,0.001 \mathrm{GHz} \leq \omega \leq 7 \mathrm{GHz}$

$$
|\mathrm{S} 12| \leq 0.0025,11.65 \mathrm{GHz} \leq \omega \leq 11.7 \mathrm{GHz}
$$

The filter structure is made of a perfect conductor on the top of a substrate with a relative dielectric constant of 10 and a height of $635 \mu \mathrm{m}$, backed with a perfect conductor ground plane. When designing a coarse model in ADS, its parameters could be tunable. This tuning capability allows one to graphically see how the parameters affect the responses. As a result, design parameters for the design procedure and parameter extraction step can appropriately be chosen. We set $L_{4}=1693 \mu \mathrm{m}, L_{5}=$ $2403 \mu \mathrm{m}$ and $L_{8}=18 \mu \mathrm{m}$ because ADS tuning process shows that these parameters do not have significant effects on design specifications. Now, the design parameters and auxiliary parameters are given by $x_{f}=$ $\left[W_{1}, W_{2}, W_{3}, W_{4}, L_{1}, L_{2}, L_{3}, L_{6}, L_{7}\right]$ and $\mathrm{p}=\left[h_{1}, h_{2}, h_{3}, h_{4}, \varepsilon_{r 1}\right.$, $\left.\varepsilon_{r 2}, \varepsilon_{r 3}, \varepsilon_{r 4}\right]$, respectively, where $h_{i}$ and $\epsilon_{r i}$ refer to the height and relative dielectric constant for each microstrip line having a width of $W_{i}$.

In the parameter extraction step, we use ADS quasi-Newton optimization algorithm to match the fine and surrogate models' magnitude of scattering parameters. The optimal coarse model is obtained using the ADS gradient optimization algorithm. The main advantage of implicit space mapping optimization technique is that, in this example, the design algorithm requires only one iteration, i.e., two fine model simulations. The coarse and fine models' responses for the initial and final design parameters are demonstrated in Figure 3 and Figure 4, respectively.

Table 1 shows the initial and final values of design parameters. The original and final values of auxiliary parameters are given in Table 2.

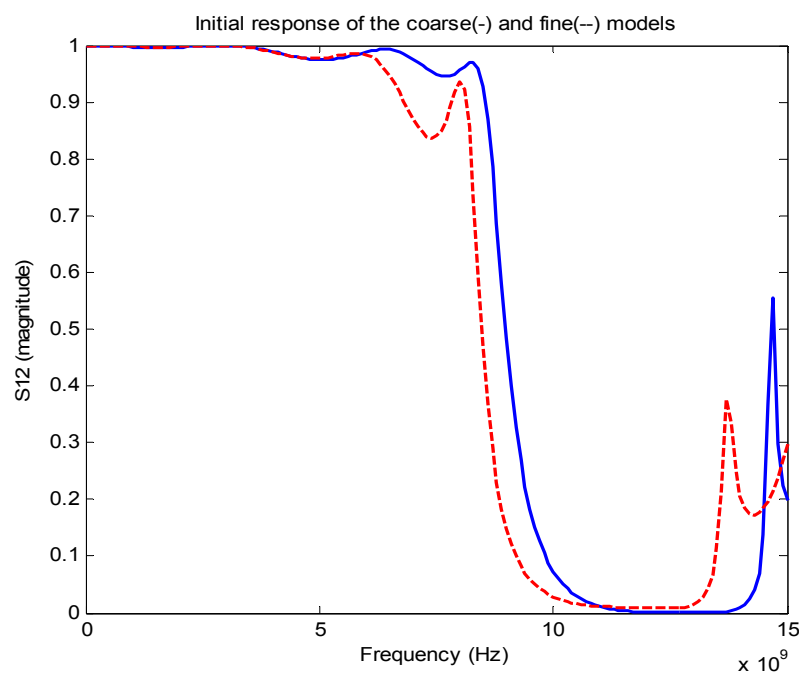

Figure 3. Coarse and fine models' responses for initial solutions.

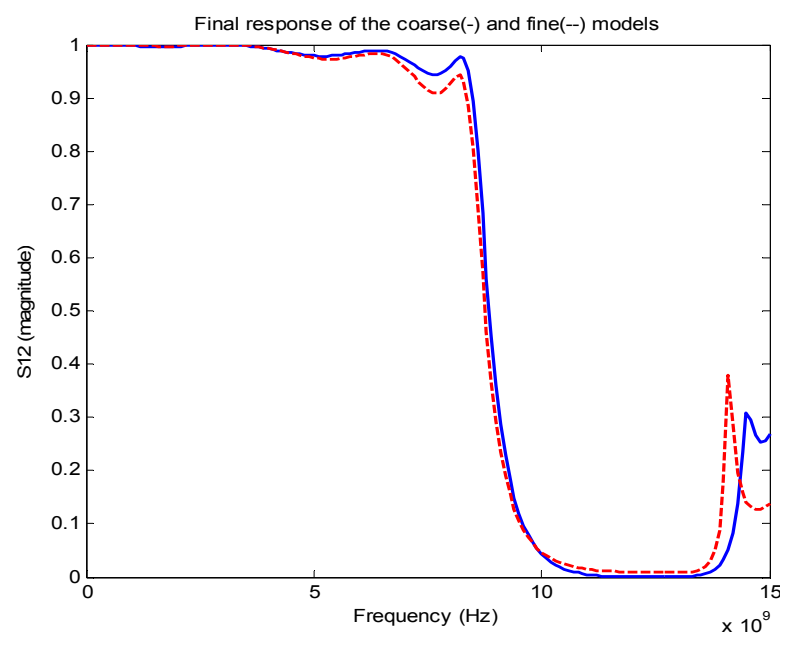

Figure 4. Coarse and fine models' responses for final solutions.

Table 1. Design parameters.

\begin{tabular}{ccc}
\hline Design parameters & Initial values & Final values \\
\hline$W_{1}(\mu \mathrm{m})$ & 638.215 & 559.408 \\
$W_{2}(\mu \mathrm{m})$ & 421.246 & 437.354 \\
$W_{3}(\mu \mathrm{m})$ & 351.031 & 336.444 \\
$W_{4}(\mu \mathrm{m})$ & 333.715 & 343.167 \\
$L_{1}(\mu \mathrm{m})$ & 690.834 & 647.674 \\
$L_{2}(\mu \mathrm{m})$ & 1536.79 & 1443.19 \\
$L_{3}(\mu \mathrm{m})$ & 811.063 & 820.294 \\
$L_{6}(\mu \mathrm{m})$ & 1646.5 & 1705.57 \\
$L_{7}(\mu \mathrm{m})$ & 774.116 & 766.248 \\
\hline
\end{tabular}

Table 2. Auxiliary parameters.

\begin{tabular}{ccc}
\hline Auxiliary parameters & Original values & Final values \\
\hline$h_{1}(\mu \mathrm{m})$ & 635 & 610.787 \\
$h_{2}(\mu \mathrm{m})$ & 635 & 764.68 \\
$h_{3}(\mu \mathrm{m})$ & 635 & 625.413 \\
$h_{4}(\mu \mathrm{m})$ & 635 & 781.898 \\
$\varepsilon_{r 1}$ & 10 & 10.3323 \\
$\varepsilon_{r 2}$ & 10 & 10.8587 \\
$\varepsilon_{r 3}$ & 10 & 10.6504 \\
$\varepsilon_{r 4}$ & 10 & 11.6629 \\
\hline
\end{tabular}

\section{Conclusions}

Using implicit space mapping method, the design parameters for a microstrip low-pass elliptic filter were determined. It was shown that this technique led to de- 
creasing the number of fine model evaluations. First, the coarse model was optimized to obtain design parameters satisfying the design objective. Second, auxiliary parameters were calibrated in the coarse model to match coarse and fine models' responses. Third, the improved coarse model was re-optimized to obtain a new set of design parameters. Finally, the resulting design parameters were given to the fine model to evaluate its performance. The design procedure was repeated by the time a satisfactory solution was obtained. Simulation results showed that only two evaluations of the fine model were sufficient to get satisfactory results for the given case-study application.

\section{References}

[1] J. W. Bandler, R. M. Biernacki, S. H. Chen, P. A. Grobelny and R. H. Hemmers, "Space Mapping Technique for Electromagnetic Optimization," IEEE Transactions on Microwave Theory and Techniques, Vol. 42, No. 12, 1994, pp. 2536-2544,.

[2] J. W. Bandler, Q. S. Cheng, S. A. Dakroury, A. S. Mohamed, M. H. Bakr, K. Madsen and J. Søndergaard, "Space Mapping: the State of the Art," IEEE Transac- tions on Microwave Theory and Techniques, Vol. 52, No. 1, 2004, pp. 337-361.

[3] J. W. Bandler, Q. S. Cheng, N. K. Nikolova and M. A. Ismail, "Implicit Space Mapping Optimization Exploiting Preassigned Parameters," IEEE Transactions on Microwave Theory and Techniques, Vol. 52, No. 1, 2004, pp. 378-385.

[4] S. Koziel, Q. S. Cheng and J. W. Bandler, "Space Mapping," IEEE Microwave Magazine, Vol. 9, No. 6, December 2008, pp. 105-122.

[5] “Agilent Advanced Design System (ADS)," Ver. 2008A, Agilent Technologies, Santa Rosa, CA, 2008.

[6] J. W. Bandler, Q. S. Cheng, D. H. Gebre-Mariam, K. Madsen, F. Pedersen and J. Søndergaard, "EM-based SurRogate Modeling and Design Exploiting Implicit, Frequency and Output Space Mappings," IEEE MTT-S International Microwave Symposium Digest, Philadelphia, 2003, pp. 1003-1006.

[7] M. C. V. Ahumada, J. Martel and F. Medina, "Design of Compact Low-Pass Elliptic Filters Using Double-Sided MIC Technology," IEEE Transactions on Microwave Theory and Techniques, Vol. 55, No. 1, January 2007, pp. 121-127. 